Canad. Math. Bull. Vol. 43 (3), 2000 pp. 343-354

\title{
Controlled Homeomorphisms Over Nonpositively Curved Manifolds
}

\author{
Bruce Hughes, Larry Taylor and Bruce Williams
}

Abstract. We obtain a homotopy splitting of the forget control map for controlled homeomorphisms over closed manifolds of nonpositive curvature.

\section{Introduction}

The theme of controlled topology is to take standard objects in geometric and algebraic topology, such as homotopy equivalences and $h$-cobordisms, and introduce estimates on the size of these objects measured via a map to an auxiliary metric space. There are many implications for classical topology and geometry-see Weinberger [11] for a recent exposition.

In this paper we study controlled homeomorphisms when the auxiliary space is a closed manifold of nonpositive curvature (i.e., a closed Riemannian manifold whose sectional curvatures are all less than or equal to zero) and the map is a fibre bundle projection (or, more generally, a manifold approximate fibration) defined on a closed manifold.

To illustrate our main result, let $h: F \rightarrow F$ be a homeomorphism on a closed manifold $F$ and let $B$ be a closed manifold of nonpositive curvature. Suppose $h \times \mathrm{id}_{B}$ is isotopic to $\mathrm{id}_{F \times B}$. Since $h$ need not be isotopic to $\mathrm{id}_{F}$, the isotopy $h \times \mathrm{id}_{B} \simeq \mathrm{id}_{F \times B}$ need not be fibre preserving over $B$; that is, when the isotopy is composed with projection to $B \times I$, the result might differ from the projection. However, we prove (assuming $\operatorname{dim} F+\operatorname{dim} B \geq 5$ ) that if the composition (of the isotopy with projection) is at least homotopic to the projection rel $F \times B \times \partial I$, then we can choose isotopies which are arbitrarily close to being fibre preserving; that is, for every $\epsilon>0$ there exists an isotopy $H^{\epsilon}$ from $h \times \mathrm{id}_{B}$ to $\mathrm{id}_{F \times B}$ such that $p_{B} H^{\epsilon}(x, y, t)$ is within $\epsilon$ of $y$ for each $(x, y, t) \in F \times B \times I$ where $p_{B}$ denotes projection to $B$ (see Corollary 5.2 for a precise statement and see Example 5.3 for homeomorphisms $h$ to which this result applies). Because close maps are homotopic, this $\epsilon$ condition is obviously sufficient for having an isotopy as above whose composition with projection is homotopic to the projection $\mathrm{rel} F \times B \times \partial I$.

This follows from a general result about the injectivity of a 'forget control' map from the space of controlled homeomorphisms to the space of homotopically controlled homeomorphisms. We work in the generality of a manifold approximate fibration $p: M \rightarrow B$ (see [5]), but our results are new and interesting for the special case of a fibre bundle projection $p: M \rightarrow B$ (or even, as in the paragraph above, for a product projection $F \times B \rightarrow B$ ).

Received by the editors July 2, 1998; revised October 4, 1999.

The first author was supported in part by NSF Grant DMS-9504759. The second and third authors were supported in part by NSF Grant DMS-9505024.

AMS subject classification: Primary: 57N15, 53C20; secondary: 55R65, 57N37.

Keywords: controlled topology, controlled homeomorphism, nonpositive curvature, Novikov conjectures.

(c) Canadian Mathematical Society 2000. 
The spaces of controlled and homotopically controlled homeomorphisms are denoted by $\operatorname{TOP}^{c}(p: M \rightarrow B)$ and $\operatorname{TOP}^{h}(p: M \rightarrow B)$, respectively (see Sections 2, 3 for the precise definitions as simplicial sets). A vertex of $\operatorname{TOP}^{h}(p: M \rightarrow B)$, a homotopically controlled homeomorphism, is a homeomorphism $h: M \rightarrow M$ together with a homotopy $p h \simeq p$. This space fits into a fibration sequence

$$
\Omega \operatorname{Map}(M, B) \longrightarrow \operatorname{TOP}^{h}(p: M \rightarrow B) \stackrel{\text { forget }}{\longrightarrow} \operatorname{TOP}(M)
$$

where the forget map forgets the homotopy (of course, the image of this map need not meet all components of $\operatorname{TOP}(M))$ and $\Omega \operatorname{Map}(M, B)$ is the loop space of the space of all maps from $M$ to $B$ based at $p: M \rightarrow B$.

A vertex of $\operatorname{TOP}^{c}(p: M \rightarrow B)$, a controlled homeomorphism, is a homeomorphism $h: M \rightarrow M$ together with a parametrized family of homeomorphisms $\left\{h_{t} \mid 0 \leq t<1\right\}$ such that $h=h_{0}$ and $\lim _{t \rightarrow 1} p h_{t}=p$. The family induces a homotopy $p h \simeq p$ so that there is a 'forget control' map $\psi: \operatorname{TOP}^{c}(p: M \rightarrow B) \rightarrow \operatorname{TOP}^{h}(p: M \rightarrow B)$.

Main Theorem If $p: M \rightarrow B$ is a manifold approximate fibration, $\operatorname{dim} M \geq 5$, and $B$ is a closed manifold of nonpositive curvature, then the forget control map

$$
\psi: \operatorname{TOP}^{c}(p: M \rightarrow B) \rightarrow \operatorname{TOP}^{h}(p: M \rightarrow B)
$$

is homotopy split injective. That is, there exists a simplicial map

$$
r: \operatorname{TOP}^{h}(p: M \rightarrow B) \rightarrow \operatorname{TOP}^{c}(p: M \rightarrow B)
$$

such that $r \circ \psi$ is homotopic to $\mathrm{id}_{\mathrm{TOP}^{c}(p: M \rightarrow B)}$.

This is restated and proved as Theorem 5.1 below.

Two special cases help illustrate the spaces of homeomorphisms involved (although the theorem is trivial in these two cases). First, if $B$ is a point, then

$$
\operatorname{TOP}^{c}(M \rightarrow \text { point }) \simeq \operatorname{TOP}^{h}(M \rightarrow \text { point }) \simeq \operatorname{TOP}(M)
$$

Second, if $M=B$ and $p=\mathrm{id}_{M}$, then $\operatorname{TOP}^{c}\left(\mathrm{id}_{M}: M \rightarrow M\right)$ is contractible (it is essentially the space of paths in $\operatorname{TOP}(M)$ based at $\left.\mathrm{id}_{M}\right)$ and there is a fibration sequence

$$
\Omega \operatorname{Map}(M, M) \longrightarrow \operatorname{TOP}^{h}\left(\mathrm{id}_{M}: M \rightarrow M\right) \longrightarrow \operatorname{TOP}(M) .
$$

The components of $\operatorname{TOP}(M)$ in the image of this fibration consist of those homeomorphisms which are homotopic to $\mathrm{id}_{M}$. When $M=B$ is a closed manifold of nonpositive curvature these components are not contractible (see Farrell and Jones [2] for explicit calculations).

When $M$ is assumed to be aspherical or, even stronger, nonpositively curved then more explicit information about controlled, and homotopically controlled, homeomorphisms is available. See Example 2.1 and Corollaries 5.4 and 5.5.

This paper is part of a series dealing with controlled topology over manifolds of nonpositive curvature [6], [7]. Future installments will deal with controlled homotopy-topological structures, controlled unstable $L$-theory, and generalized Novikov conjectures for surgery and concordance theory [8], [9]. 


\section{Delooping Homotopically Controlled Homeomorphisms}

The goal of this section is to define $\operatorname{TOP}^{h}(p: M \rightarrow B)$ and show that it is homotopy equivalent to the loop space of a certain simplicial set $\operatorname{Spaces}(B)$ which we will define below.

Let $B$ be a topological space. Let $M$ be a finite dimensional, separable metric space with a fixed closed embedding $i: M \rightarrow \ell_{2}$ such that for some positive integer $N, i(M)$ is a subspace of the standard $\mathbb{R}^{N} \subseteq \ell_{2}$. For notational simplicity, we will also let $i$ denote the embedding $i \times \operatorname{id}_{\Delta^{k}}: M \times \Delta^{k} \rightarrow \ell_{2} \times \Delta^{k}$.

\section{Homeomorphisms and Maps}

Let $\operatorname{TOP}(M)$ be the simplicial group of homeomorphisms on $M$. Thus, a $k$-simplex is a homeomorphism $h: M \times \Delta^{k} \rightarrow M \times \Delta^{k}$ which is fibre preserving over $\Delta^{k}$.

Let $\operatorname{Map}(M, B)$ be the simplicial set of maps from $M$ to $B$. Thus, a $k$-simplex is a map $f: M \times \Delta^{k} \rightarrow B \times \Delta^{k}$ which is fibre preserving over $\Delta^{k}$. The fibre preserving condition is a matter of convenience for this particular simplicial set: $\operatorname{Map}(M, B)$ as we have defined it, is homotopy equivalent to the similarly defined simplicial set with a blocked (instead of fibre preserving) condition.

Fix a map $p: M \rightarrow B$. Thus, $p$ is a vertex in $\operatorname{Map}(M, B)$. Define a simplicial map $\Psi: \operatorname{TOP}(M) \rightarrow \operatorname{Map}(M, B)$ by

$$
\Psi(h)=\left(p \times \operatorname{id}_{\Delta^{k}}\right) \circ h: M \times \Delta^{k} \rightarrow B \times \Delta^{k} .
$$

Our convention for the standard simplices is that $\Delta^{k+1}$ is the span $\left\langle v_{0}, \ldots, v_{k+1}\right\rangle$ and the $k+1$-face is $\partial_{k+1} \Delta^{k+1}=\left\langle v_{0}, \ldots, v_{k}\right\rangle=\Delta^{k}$.

\section{Homotopically Controlled Homeomorphisms}

Let $\operatorname{TOP}^{h}(p: M \rightarrow B)=\operatorname{TOP}^{h}(p)$ denote the homotopy fibre of $\Psi$ over $p$. Thus, a $k$ simplex of $\operatorname{TOP}^{h}(p)$ consists of an ordered pair $(h, g)$ where $h: M \times \Delta^{k} \rightarrow M \times \Delta^{k}$ is a $k$-simplex of TOP $(M)$ and $g: M \times \Delta^{k+1} \rightarrow B \times \Delta^{k+1}$ is a $(k+1)$-simplex of $\operatorname{Map}(M, B)$ such that

1) $g \mid\left(M \times v_{k+1}\right)=p$, and

2) $g \mid\left(M \times \partial_{k+1} \Delta^{k+1}\right)=\left(p \times \operatorname{id}_{\Delta^{k}}\right) \circ h$.

For example, a vertex of $\mathrm{TOP}^{h}(p)$ essentially consists of a homeomorphism $h: M \rightarrow M$ together with a homotopy from $p h$ to $p$. This is why we call the elements of $\operatorname{TOP}^{h}(p)$ homotopically controlled.

The fibration $\operatorname{TOP}^{h}(p: M \rightarrow B) \rightarrow \operatorname{TOP}(M) \stackrel{\Psi}{\longrightarrow} \operatorname{Map}(M, B)$ induces a long exact sequence of homotopy groups:

$$
\begin{aligned}
\cdots & \rightarrow \pi_{n+1} \operatorname{Map}(M, B) \rightarrow \pi_{n} \operatorname{TOP}^{h}(p: M \rightarrow B) \rightarrow \pi_{n} \operatorname{TOP}(M) \rightarrow \cdots \\
& \rightarrow \pi_{1} \operatorname{Map}(M, B) \rightarrow \pi_{0} \operatorname{TOP}^{h}(p: M \rightarrow B) \rightarrow \pi_{0} \operatorname{TOP}(M) \rightarrow \pi_{0} \operatorname{Map}(M, B) .
\end{aligned}
$$


Example 2.1 Let $p: M \rightarrow B$ be a map with $M$ and $B$ (connected and locally compact) aspherical ANRs. Fix basepoints in $M$ and $B$ and let $\operatorname{Map}_{*}(M, B)$ be the simplicial set of based maps so that there is a fibration

$$
\operatorname{Map}_{*}(M, B) \stackrel{\text { inclusion }}{\longrightarrow} \operatorname{Map}(M, B) \stackrel{\text { evaluation }}{\longrightarrow} B
$$

Since $\pi_{n}(B)$ vanishes for $n \neq 1$ and $\operatorname{Map}_{*}(M, B)$ is homotopy equivalent to the discrete set of group homomorphisms $\operatorname{hom}\left(\pi_{1} M, \pi_{1} B\right)$, it follows from this fibration that $\pi_{n} \operatorname{Map}(M, B)=0$ for $n \geq 2$. Thus, the induced forgetful homomorphism

$$
\pi_{n} \operatorname{TOP}^{h}(p: M \rightarrow B) \longrightarrow \pi_{n} \operatorname{TOP}(M)
$$

is an isomorphism for $n \geq 2$, and an injection for $n=1$. See Corollaries 5.4 and 5.5 for an application.

\section{Spaces}

Let Spaces $(B)$ be the simplicial set of spaces over $B$. A $k$-simplex consists of a closed subset $X$ of $\mathbb{R}^{L} \times B \times \Delta^{k} \subseteq \ell_{2} \times B \times \Delta^{k}$ for some $L$ depending on $X$, such that the composition

$$
X \subseteq \ell_{2} \times B \times \Delta^{k} \stackrel{\text { proj }}{\longrightarrow} \Delta^{k}
$$

is the projection of a locally trivial fibre bundle. The restriction of the projection $\ell_{2} \times B \times$ $\Delta^{k} \rightarrow B \times \Delta^{k}$ defines a map $X \rightarrow B \times \Delta^{k}$, so a $k$-simplex is essentially a $k$-parameter family of spaces over $B$.

There is a closed embedding $j: M \rightarrow \ell_{2} \times B$ defined by $x \mapsto(i(x), p(x))$. In this way, $p: M \rightarrow B$ determines a vertex of $\operatorname{Spaces}(B)$ which we will denote by $p$. We will also use $j$ to denote the embedding $j \times \mathrm{id}_{\Delta^{k}}$.

\section{Delooping}

We will now define a simplicial map $\Gamma: \operatorname{TOP}^{h}(p) \rightarrow \Omega$ Spaces $(B)$ where the loops are based at $p$, and then prove that $\Gamma$ is a homotopy equivalence. We begin with the following version of the well-known Klee trick (see e.g. [10, p. 74]).

For any space $Y$, let Iso $(Y)$ denote the simplicial set of isotopies on $Y$. Thus, a $k$-simplex is a homeomorphism $h: Y \times \Delta^{k} \times I \rightarrow Y \times \Delta^{k} \times I$ which is fibre preserving over $\Delta^{k} \times I$ and $h \mid Y \times \Delta^{k} \times\{0\}=\operatorname{id}_{Y \times \Delta^{k} \times\{0\}}$. Recall $N$ is an integer such that $i(M) \subseteq \mathbb{R}^{N}$. The following proposition says that every homeomorphism of $M$ can be realized by an isotopy of $\mathbb{R}^{2 N}$.

Proposition 2.2 There exists a simplicial map $\alpha: \operatorname{TOP}(M) \rightarrow \operatorname{Iso}\left(\mathbb{R}^{2 N}\right)$ such that for each $k$-simplex $h$ of $\mathrm{TOP}(M)$,

$$
\alpha(h)_{1} \circ i \circ h=i
$$

where $i$ denotes the embedding $i \times \operatorname{id}_{\Delta^{k}}: M \times \Delta^{k} \rightarrow \mathbb{R}^{N} \times \Delta^{k}$. 
Proof Consider the graph of $i h i^{-1}: i(M) \rightarrow i(M)$ as a subset of $\mathbb{R}^{2 N}$. As usual, push up to this graph, over to the image, then back over to the diagonal and down to the domain.

Given a $k$-simplex $(h, g)$ of $\operatorname{TOP}^{h}(p)$, we will describe a closed embedding

$$
\beta(h, g)=\beta: M \times \Delta^{k+1} \rightarrow \ell_{2} \times B \times \Delta^{k+1}
$$

and then define $\Gamma((h, g))$ to be the image of $\beta$.

For $u \in \Delta^{k+1}$, write $u=t v_{k+1}+(1-t) w$ where $w \in \Delta^{k}$ and $0 \leq t \leq 1$. Then for $x \in M$, define

$$
\beta(x, u)=\left(\operatorname{proj}_{\ell_{2}} \circ \alpha(h)_{t} \circ i \circ h(x, w), g(x, u)\right) .
$$

Note that

$$
\beta \mid M \times \Delta^{k}=j h \quad \text { and } \quad \beta \mid M \times v_{k+1}=j .
$$

Lemma 2.3 $\beta: M \times \Delta^{k+1} \rightarrow \ell_{2} \times B \times \Delta^{k+1}$ is a closed embedding.

Proof Consider the composition

$$
\beta^{\prime}: M \times \Delta^{k+1} \stackrel{\beta}{\longrightarrow} \ell_{2} \times B \times \Delta^{k+1} \stackrel{\text { proj }}{\longrightarrow} \ell_{2} \times \Delta^{k+1} .
$$

We begin by showing that $\beta^{\prime}$ is a closed embedding.

To this end, let $q: \Delta^{k} \times I \rightarrow \Delta^{k+1}$ be the quotient map given by $(w, t) \mapsto t v_{k+1}+(1-t) w$. Define $\beta^{\prime \prime}$ to be the composition

$$
\beta^{\prime \prime}: M \times \Delta^{k} \times I \stackrel{h \times \mathrm{id}_{I}}{\longrightarrow} M \times \Delta^{k} \times I \stackrel{i \times \mathrm{id}_{I}}{\longrightarrow} \ell_{2} \times \Delta^{k} \times I \stackrel{\alpha(h)}{\longrightarrow} \ell_{2} \times \Delta^{k} \times I .
$$

Note that $\beta^{\prime \prime}$ is a closed embedding. The usual transgression lemma then implies that $\beta^{\prime}$ is an embedding.

Now note that $\left(\operatorname{id}_{M} \times q\right) \beta^{\prime \prime}=\beta^{\prime}\left(\operatorname{id}_{M} \times q\right)$. Since $\Delta^{k} \times I$ is compact, it follows that $\operatorname{id}_{M} \times q$ and, hence $\left(\operatorname{id}_{M} \times q\right) \beta^{\prime \prime}$, are closed maps. Also, since $\beta^{\prime \prime} \mid M \times \Delta^{k} \times 1=i=$ $\beta^{\prime} \mid M \times v_{k+1}$, it follows that $\operatorname{Im}\left(\beta^{\prime}\right)=\operatorname{Im}\left(\left(\operatorname{id}_{M} \times q\right) \circ \beta^{\prime \prime}\right)$. Hence, $\operatorname{Im}\left(\beta^{\prime}\right)$ is closed in $\ell_{2} \times \Delta^{k+1}$ and $\beta^{\prime}$ is a closed embedding.

In order to see that $\beta$ itself is an embedding, let $\gamma: \operatorname{Im}\left(\beta^{\prime}\right) \rightarrow M \times \Delta^{k+1}$ be the (continuous) inverse for $\beta^{\prime}$. Then it is easy to check that the composition

$$
\operatorname{Im}(\beta) \stackrel{\text { proj }}{\longrightarrow} \operatorname{Im}\left(\beta^{\prime}\right) \stackrel{\gamma}{\longrightarrow} M \times \Delta^{k+1}
$$

is an inverse for $\beta$.

Finally, to see that $\operatorname{Im}(\beta)$ is closed in $\ell_{2} \times B \times \Delta^{k+1}$, consider the composition

$$
g^{\prime}: M \times \Delta^{k+1} \stackrel{g}{\longrightarrow} B \times \Delta^{k+1} \stackrel{\text { proj }}{\longrightarrow} B .
$$

Then, up to permutation of coordinates, $\beta$ is given by $x \mapsto\left(\beta^{\prime}(x), g^{\prime}(x)\right)$. Therefore, $\operatorname{Im}(\beta)$ is essentially the graph of a map $\left(g^{\prime}\right)$ from a closed subset $\left(\operatorname{Im}\left(\beta^{\prime}\right)\right)$ of $\ell_{2} \times \Delta^{k+1}$ to $B$. Hence, $\operatorname{Im}(\beta)$ is closed.

Lemma 2.4 $\operatorname{Im}(\beta) \subseteq \ell_{2} \times B \times \Delta^{k+1}$ is a $k$-simplex of $\Omega$ Spaces $(B)$ where the loops are based at $p$. 
Proof Let $W=\operatorname{Im}(\beta)$. We first need to observe that $W$ is a $(k+1)$-simplex of Spaces $(B)$. It follows from 2.3 and the definition of $\beta$ that $W$ is closed in $\mathbb{R}^{2 N} \times B \times \Delta^{k+1}$. Moreover, since $\beta$ is fibre preserving over $\Delta^{k+1}$ and $\beta$ is an embedding, it follows that proj $\mid: W \rightarrow \Delta^{k+1}$ is a bundle projection.

We also have to check that $W$ is a loop in $\operatorname{Spaces}(B)$ based at $p$. For this we have to look at the vertex $\partial_{0}^{k+1} W$ and the face $\partial_{k+1} W$. In the first case, we have $\partial_{0}^{k+1} W=W \cap \ell_{2} \times B \times v_{k+1}=$ $\beta\left(M \times v_{k+1}\right)$. Since $\beta \mid M \times v_{k+1}=j$, it follows that $\partial_{0}^{k+1} W$ is the basepoint $p$ of $\operatorname{Spaces}(B)$.

Likewise, it is easy to check that the face $\partial_{k+1} W=W \cap \ell_{2} \times B \times \Delta^{k}=\beta\left(M \times \Delta^{k}\right)$ equals $j\left(M \times \Delta^{k}\right)$ which is the base $k$-simplex of $\operatorname{Spaces}(B)$.

Theorem 2.5 $\Gamma: \operatorname{TOP}^{h}(p) \rightarrow \Omega \operatorname{Spaces}(B)$, defined by $\Gamma(h, g)=\operatorname{Im}(\beta(h, g))$, is a homotopy equivalence.

Proof It is straightforward to check that $\Gamma$ is a simplicial map. Moreover, $\operatorname{TOP}^{h}(p)$ and Spaces $(B)$ satisfy the Kan condition. Hence, it suffices to show that $\Gamma$ induces an isomorphism on homotopy groups (and a bijection between components). To this end, suppose we have a $k$-simplex $X \subseteq \ell_{2} \times B \times \Delta^{k+1}$ of $\Omega$ Spaces $(B), k \geq 0$, such that each of the faces of $X$ making up the boundary $\partial X \subseteq \ell_{2} \times B \times \partial \Lambda^{k+1}$ is in the image of $\Gamma$. Here $\Lambda^{k+1}$ is the horn $\partial \Delta^{k+1}-\operatorname{int} \Delta^{k}$. After amalgamating the various $(k-1)$-simplices of $\operatorname{TOP}^{h}(p)$ which map to $\partial X$, we have $(h, g)$ with $\Gamma(h, g)=\partial X$ where $h: M \times \partial \Delta^{k} \rightarrow M \times \partial \Delta^{k}$ is a homeomorphism (fibre preserving over $\Delta^{k}$ ) and $g: M \times \Lambda^{k+1} \rightarrow B \times \Lambda^{k+1}$ is a map such that $g \mid M \times v_{k+1}=p$ and $g \mid M \times \partial \Delta^{k}=\left(p \times \operatorname{id}_{\partial \Delta^{k}}\right) \circ h$.

We need a $k$-simplex $(\tilde{h}, \tilde{g})$ of $\operatorname{TOP}^{h}(p)$ such that $\partial(\tilde{h}, \tilde{g})=(h, g)$ and $\Gamma(\tilde{h}, \tilde{g}) \simeq X$ rel $\partial X$. The definition of $\Gamma$ implies that the pair $(h, g)$ gives rise to a closed embedding $\beta: M \times \Lambda^{k+1} \rightarrow \ell_{2} \times B \times \Lambda^{k+1}$ such that $\operatorname{Im}(\beta)=\partial X$. Recall that proj $\mid: X \rightarrow \Delta^{k+1}$ is a bundle projection, and note that $\beta$ is a partial trivialization of this bundle. It follows that there is a homeomorphism $\tilde{\beta}: M \times \Delta^{k+1} \rightarrow X$ which is fibre preserving over $\Delta^{k+1}$ and $\tilde{\beta} \mid M \times \Lambda^{k+1}=\beta$. Define $\tilde{h}$ to be the composition

$$
M \times \Delta^{k} \stackrel{\tilde{\beta} \mid}{\longrightarrow} j\left(M \times \Delta^{k}\right) \stackrel{j^{-1}}{\longrightarrow} M \times \Delta^{k} .
$$

Define $\tilde{g}$ to be the composition

$$
M \times \Delta^{k+1} \stackrel{\tilde{\beta}}{\longrightarrow} X \stackrel{\text { proj }}{\longrightarrow} B .
$$

Clearly, $(\tilde{h}, \tilde{g})$ is a $k$-simplex of $\operatorname{TOP}^{h}(p)$ such that $\partial(\tilde{h}, \tilde{g})=(h, g)$. So it remains to show that $\Gamma(\tilde{h}, \tilde{g}) \simeq X \operatorname{rel} \partial X$.

The definition of $\Gamma$ implies that the $k$-simplex $(\tilde{h}, \tilde{g})$ gives rise to a closed embedding $\beta^{*}: M \times \Delta^{k+1} \rightarrow \mathbb{R}^{N} \times B \times \Delta^{k+1} \subseteq \ell_{2} \times B \times \Delta^{k+1}$, some $N$. Moreover, $\beta^{*} \mid M \times \partial \Delta^{k+1}=$ $\tilde{\beta} \mid$. Now an elementary construction provides a homotopy from $\beta^{*}$ to $\tilde{\beta}$ through closed embeddings into $\mathbb{R}^{L} \times B \times \Delta^{k+1}$, some $L$, with the homotopy being fibre preserving over $\Delta^{k+1}$ and rel $M \times \partial \Delta^{k+1}$. (Start with any homotopy $\hat{\beta}: M \times \Delta^{k+1} \times I \rightarrow \mathbb{R}^{L} \times B \times \Delta^{k+1}$ from $\beta^{*}$ to $\tilde{\beta}$ which is fibre preserving over $\Delta^{k+1}$ and rel $\partial \Delta^{k+1}$. Then define $\bar{\beta}: M \times \Delta^{k+1} \times I \rightarrow$ $\mathbb{R}^{L} \times B \times \Delta^{k+1} \times \mathbb{R}^{L}$ by

$$
(x, u, t) \longmapsto(\hat{\beta}(x, u, t), t(1-t) \rho(u) i(x))
$$


where $\rho: \Delta^{k+1} \rightarrow \mathbb{R}$ is any map satisfying $\rho^{-1}(0)=\partial \Delta^{k+1}$. The desired homotopy comes from permuting the components of $\bar{\beta}$.) This homotopy provides a homotopy in $\Omega \operatorname{Spaces}(B)$ from $\Gamma(\tilde{h}, \tilde{g})$ to $X \operatorname{rel} \partial X$.

\section{Controlled Homeomorphisms and the Forget Control Map}

We use the same notation as in the previous section, so that in particular we have fixed a map $p: M \rightarrow B$ for spaces $M$ and $B$ and a closed embedding $i: M \rightarrow \mathbb{R}^{N} \subseteq \ell_{2}$, some $N$.

\section{Controlled Homeomorphisms}

The simplicial group $\operatorname{TOP}^{c}(p: M \rightarrow B)=\operatorname{TOP}^{c}(p)$ of controlled homeomorphisms on $p$ was defined in [5]. A $k$-simplex is a homeomorphism

$$
h: M \times \Delta^{k} \times[0,1) \longrightarrow M \times \Delta^{k} \times[0,1)
$$

which is fibre preserving over $\Delta^{k} \times[0,1)$ and such that the compositions

$$
\begin{gathered}
M \times \Delta^{k} \times[0,1) \stackrel{h}{\longrightarrow} M \times \Delta^{k} \times[0,1) \stackrel{p \times \text { id }}{\longrightarrow} B \times \Delta^{k} \times[0,1), \text { and } \\
M \times \Delta^{k} \times[0,1) \stackrel{h^{-1}}{\longrightarrow} M \times \Delta^{k} \times[0,1) \stackrel{p \times \text { id }}{\longrightarrow} B \times \Delta^{k} \times[0,1)
\end{gathered}
$$

extend to continuous maps

$$
M \times \Delta^{k} \times[0,1] \longrightarrow B \times \Delta^{k} \times[0,1]
$$

via $p \times$ id: $M \times \Delta^{k} \times 1 \rightarrow B \times \Delta^{k} \times 1$.

There is a forget control map $\psi: \operatorname{TOP}^{c}(p) \rightarrow \operatorname{TOP}^{h}(p)$ defined as follows. First define $q: \Delta^{k} \times[0,1) \rightarrow \Delta^{k+1}$ by $(w, t) \mapsto t v_{k+1}+(1-t) w$ so that $q$ is the restriction of the map called $q$ in the proof of 2.3. Now given a $k$-simplex $h: M \times \Delta^{k} \times[0,1) \rightarrow M \times \Delta^{k} \times[0,1)$, define a map $\widehat{p h}: M \times \Delta^{k+1} \rightarrow B \times \Delta^{k+1}$ by

$$
\begin{cases}(x, u) \longmapsto(p \times q) h\left(x, q^{-1}(u)\right), & \text { if } u \neq v_{k+1} \\ \left(x, v_{k+1}\right) \longmapsto\left(p(x), v_{k+1}\right), & \text { otherwise. }\end{cases}
$$

Finally, define $\psi(h)=\left(h_{0}, \widehat{p h}\right)$ where $h_{0}=h \mid M \times \Delta^{k} \times 0$. One can check that $\psi$ is a simplicial map.

\section{Manifold Approximate Fibrations and Manifolds}

Now assume that $B$ is a (topological) manifold without boundary and fix a positive integer $m$. The simplicial set $\operatorname{MAF}(B)$ of manifold approximate fibrations over $B$ was defined in [5]. A $k$-simplex is a subset $W$ of $\mathbb{R}^{N} \times B \times \Delta^{k} \subseteq \ell_{2} \times B \times \Delta^{k}$, some $N$, so that the restriction of the projection defines a map $f: W \rightarrow B \times \Delta^{k}$ such that the composition

$$
W \stackrel{f}{\longrightarrow} B \times \Delta^{k} \stackrel{\text { proj }}{\longrightarrow} \Delta^{k}
$$


is a fibre bundle projection with fibres $m$-dimensional manifolds without boundary, and for each $t \in \Delta^{k}, f \mid: f^{-1}(B \times t) \rightarrow B \times t$ is a manifold approximate fibration. (Actually, in [5] $W$ was only required to be embedded in $\ell_{2} \times B \times \Delta^{k}$ as a set of small capacity. This technicality can be safely ignored for finite dimensional $W$ ).

The simplicial set $\mathrm{MAN}(B)$ of manifolds over $B$ was defined in [7]. It is the sub-simplicial set of Spaces $(B)$ consisting of those $k$-simplices $X \subseteq \ell_{2} \times B \times \Delta^{k}$ such that the fibres of $X \rightarrow \Delta^{k}$ are $m$-manifolds without boundary. Thus, $\operatorname{MAN}(B)$ is actually a union of certain components of Spaces $(B)$ and for the map $\Gamma$ of Section 2 we have the following immediate consequence of Theorem 2.5:

Corollary 3.1 $\Gamma: \operatorname{TOP}^{h}(p) \rightarrow \Omega \operatorname{MAN}(B)$ is a homotopy equivalence.

Also in [7] we defined a forget control map $\varphi: \operatorname{MAF}(B) \rightarrow \operatorname{MAN}(B)$ which indeed just forgets the fact that the maps to $B$ are manifold approximate fibrations. Of course, $\operatorname{MAF}(B)$ is a sub-simplicial set of $\mathrm{MAN}(B)$ (but not necessarily a union of components) and $\varphi$ is just the inclusion map.

Assume from now on that the fixed map $p: M \rightarrow B$ is a manifold approximate fibration so that $j(M)$ is a vertex of $\operatorname{MAF}(B)$ which we continue to denote by $p$.

Now define a simplicial map $\Lambda: \operatorname{TOP}^{c}(p) \rightarrow \Omega \operatorname{MAF}(B)$, where the loops are based at $p$, so that the following diagram commutes (on the nose):

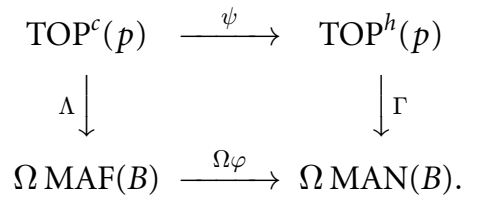

More precisely, if $h: M \times \Delta^{k} \times[0,1) \rightarrow M \times \Delta^{k} \times[0,1)$ is a $k$-simplex of $\operatorname{TOP}^{c}(p)$, then $\psi(h)=\left(h_{0}, \widehat{p h}\right)$ and the definition of $\Gamma$ gives an embedding

$$
\beta=\beta\left(h_{0}, \widehat{p h}\right): M \times \Delta^{k+1} \rightarrow \ell_{2} \times B \times \Delta^{k+1}
$$

so that $\Gamma \psi(h)=\operatorname{Im}(\beta)$. Simply note that $\operatorname{Im}(\beta)$ is in fact a $k$-simplex of $\Omega \operatorname{MAF}(B)$ and define $\Lambda(h)=\operatorname{Im}(\beta)$.

\section{Delooping Controlled Homeomorphisms}

We continue to use the notation of the previous two sections, so that in particular $p: M \rightarrow$ $B$ is a fixed manifold approximate fibration and $m=\operatorname{dim} M$.

In [5] we proved that $\operatorname{TOP}^{c}(p)$ and $\Omega \operatorname{MAF}(B)$ are homotopy equivalent (if $m \geq 5$ ), but we did not exhibit an explicit homotopy equivalence. In this section we show that the map $\Lambda$ defined in Section 3 is a homotopy equivalence. It is important to have a homotopy equivalence making diagram $(*)$ in Section 3 commute.

Theorem 4.1 If $m \geq 5$, then $\Lambda: \operatorname{TOP}^{c}(p) \rightarrow \Omega \operatorname{MAF}(B)$ is a homotopy equivalence.

Proof Both of the simplicial sets satisfy the Kan condition, so the strategy is similar to that of the proof of Theorem 2.5. So let $W \subseteq \ell_{2} \times B \times \Delta^{k+1}$ be a $k$-simplex of $\Omega \operatorname{MAF}(B)$ 
such that the faces making up $\partial W \subseteq \ell_{2} \times B \times \Lambda^{k+1}$ are in the image of $\Lambda$. Thus, after amalgamation, we have a controlled homeomorphism

$$
h: M \times \partial \Delta^{k} \times[0,1) \longrightarrow M \times \partial \Delta^{k} \times[0,1)
$$

such that $\Lambda(h)=\partial W$.

We need a $k$-simplex $\tilde{h}$ of $\operatorname{TOP}^{c}(p)$ such that $\partial \tilde{h}=h$ and $\Lambda(\tilde{h}) \simeq W$ rel $\partial W$. The constructions above yield a map $\widehat{p h}: M \times \Lambda^{k+1} \rightarrow B \times \Lambda^{k+1}$ and an embedding

$$
\beta=\beta\left(h_{0}, \widehat{p h}\right): M \times \Lambda^{k+1} \rightarrow \ell_{2} \times B \times \Lambda^{k+1}
$$

such that $\partial W=\Lambda(h)=\operatorname{Im}(\beta)$ and $\operatorname{proj} \circ \beta=\widehat{p h}$. Moreover, we have

$$
\begin{gathered}
\beta \mid M \times \partial \Delta^{k}=j h_{0}, \\
\beta \mid M \times v_{k+1}=j, \\
\widehat{p h} \mid M \times\left(\Lambda^{k+1}-v_{k+1}\right)=(p \times q) \circ h \circ\left(\operatorname{id}_{M} \times q^{-1} \mid\right), \quad \text { and } \\
\widehat{p h} \mid M \times v_{k+1}=p .
\end{gathered}
$$

Since $\beta$ is a partial trivialization of $W \rightarrow \Delta^{k+1}$, there is a homeomorphism $\tilde{\beta}: M \times \Delta^{k+1} \rightarrow$ $W$ which is fibre preserving over $\Delta^{k+1}$ and $\tilde{\beta} \mid M \times \Lambda^{k+1}=\beta$. Let $f: M \times \Delta^{k+1} \rightarrow B \times$ $\Delta^{k+1}$ be the composition proj $\circ \tilde{\beta}$. By controlled straightening [5, Section 14], there exists a homeomorphism

$$
H: M \times \Delta^{k+1} \times[0,1) \rightarrow M \times \Delta^{k+1} \times[0,1)
$$

which is a controlled homeomorphism from $p \times \operatorname{id}_{\Delta^{k+1}}$ to $f$. Moreover, we can insist that $H \mid M \times \Lambda^{k+1} \times[0,1)$ is given by

$$
\begin{cases}(x, u, t) \longmapsto(\mathrm{id} \times q) H^{-1}\left(h_{\left((1-t) q^{-1}(u)+t\right)}(x), q^{-1}(u)\right), & \text { if } u \neq v_{k+1} \\ \left(x, v_{k+1}, t\right) \longmapsto\left(x, v_{k+1}, t\right), & \text { otherwise. }\end{cases}
$$

In particular,

$$
\begin{gathered}
H \mid M \times \partial \Delta^{k} \times[0,1)=\left(h_{0}^{-1} \times \mathrm{id}\right) \circ h, \quad \text { and } \\
H \mid M \times \Lambda^{k+1} \times\{0\}=\mathrm{id} .
\end{gathered}
$$

Now let $\tilde{h}: M \times \Delta^{k} \times[0,1) \rightarrow M \times \Delta^{k} \times[0,1)$ be the composition

$M \times \Delta^{k} \times[0,1) \stackrel{H \mid}{\longrightarrow} M \times \Delta^{k} \times[0,1) \stackrel{\tilde{\beta} \mid \times \text { id }}{\longrightarrow} j\left(M \times \Delta^{k}\right) \times[0,1) \stackrel{j^{-1} \times \text { id }}{\longrightarrow} M \times \Delta^{k} \times[0,1)$.

In particular, $\tilde{h}$ is a $k$-simplex of $\operatorname{TOP}^{c}(p)$ and $\tilde{h} \mid M \times \partial \Delta^{k} \times[0,1)=h$, so that $\partial \tilde{h}=h$. The proof that $\lambda(\tilde{h}) \simeq W$ rel $\partial W$ is similar to that part of the proof of Theorem 2.5 so we omit further details. 


\section{Conclusion}

In this section we restate and prove the main theorem (Theorem 5.1) and derive some consequences.

Theorem 5.1 If $p: M \rightarrow B$ is a manifold approximate fibration, $\operatorname{dim} M \geq 5$ and $B$ is a closed manifold of nonpositive curvature, then the forget control map $\psi: \operatorname{TOP}^{c}(p) \rightarrow \operatorname{TOP}^{h}(p)$ is homotopy split injective.

Proof In [7] we showed that under these hypothesis, $\varphi: \operatorname{MAF}(B) \rightarrow \operatorname{MAN}(B)$ is homotopy split injective. The result then follows immediately from Corollary 3.1, Theorem 4.1 and the commutativity of diagram $(*)$ at the end of Section 3.

We now derive the application mentioned at the beginning of the introduction. If $f: X \rightarrow Y$ is a map, $Y$ has a specified metric and $\epsilon>0$, then an isotopy $H: X \times I \rightarrow X \times I$ is an $f^{-1}(\epsilon)$-isotopy provided $f H_{t}(x)$ is $\epsilon$-close to $f H_{0}(x)$ for every $(x, t) \in X \times I$.

Corollary 5.2 Let $h: F \rightarrow F$ be a homeomorphism on a closed manifold $F$, let $B$ be a closed manifold of nonpositive curvature such that $\operatorname{dim} F+\operatorname{dim} B \geq 5$ and let $p=\operatorname{proj}: F \times B \rightarrow B$. If $G: F \times B \times I \rightarrow F \times B \times I$ is an isotopy from $h \times \mathrm{id}_{B}$ to $\operatorname{id}_{F \times B}$ such that $\left(p \times \mathrm{id}_{I}\right) G$ is homotopic to $p \times \mathrm{id}_{I}$ rel $F \times B \times \partial I$, then for every $\epsilon>0$ there exists a $p^{-1}(\epsilon)$-isotopy $H^{\epsilon}$ from $h \times \mathrm{id}_{B}$ to $\mathrm{id}_{F \times B}$.

Proof Note that $h \times \mathrm{id}_{B \times[0,1)}$ and $\operatorname{id}_{F \times B \times[0,1)}$ are vertices of $\operatorname{TOP}^{c}(p)$. We claim that $\psi\left(h \times \mathrm{id}_{B \times[0,1)}\right)$ and $\psi\left(\mathrm{id}_{F \times B \times[0,1)}\right)$ are in the same component of $\operatorname{TOP}^{h}(p)$. For if $G: F \times$ $B \times I \rightarrow F \times B \times I$ is an isotopy from $h \times \mathrm{id}_{B}$ to $\mathrm{id}_{F \times B}$ such that $\left(p \times \mathrm{id}_{I}\right) G$ is homotopic to $p \times \mathrm{id}_{I}$, then it follows that there is a 2-simplex $J: F \times B \times \Delta^{2} \rightarrow B \times \Delta^{2}$ of $\operatorname{Map}(F \times B, B)$ such that $J \mid\left(F \times B \times \partial_{2} \Delta^{2}\right)=\left(p \times \mathrm{id}_{I}\right) G$ and $J \mid\left(F \times B \times\left(\partial \Delta^{2} \backslash \partial_{2} \Delta^{2}\right)\right)=p \times \mathrm{id}_{\partial \Delta^{2} \backslash \partial_{2} \Delta^{2}}$. Thus, $(G, J)$ is a 1 -simplex of $\operatorname{TOP}^{h}(p)$ from $\psi\left(h \times \mathrm{id}_{B \times[0,1)}\right)$ to $\psi\left(\operatorname{id}_{F \times B \times[0,1)}\right)$. It follows from Theorem 5.1 that $h \times \mathrm{id}_{B \times[0,1)}$ and $\mathrm{id}_{F \times B \times[0,1)}$ are in the same component of $\operatorname{TOP}^{c}(p)$. If $H: F \times B \times I \times[0,1) \rightarrow F \times B \times I \times[0,1)$ is a $1-$ simplex of $\operatorname{TOP}^{c}(p)$ between these two vertices (where we are identifying $I$ and $\Delta^{1}$ ) and $\epsilon>0$ is given, then $H^{\epsilon}=H \mid(F \times B \times I \times\{t\})$ is the desired $\left(p \times \mathrm{id}_{B}\right)^{-1}(\epsilon)$-isotopy for $t$ close enough to 1 .

Example 5.3 Here are some general considerations which lead to specific examples of homeomorphisms satisfying the hypotheses of Corollary 5.2. Start with any closed manifold $F$ for which there is a homeomorphism $h: F \rightarrow F$ which is concordant, but not isotopic, to the identity on $F$ (for example, Hatcher [3, Section 4] shows that there are such homeomorphisms on $n$-dimensional tori for any $n \geq 5$ ). Let $H: F \times I \rightarrow F \times I$ be a concordance from $\mathrm{id}_{F}$ to $h$; that is, $H$ is a homeomorphism such that $H \mid: F \times\{0\} \rightarrow F \times\{0\}$ is the identity and $h=H \mid: F \times\{1\} \rightarrow F \times\{1\}$. Now let $B$ be any closed nonpositively curved manifold with euler characteristic $\chi(B)=0$ (of course, this is the case for odd dimensional closed nonpositively curved manifolds). By the product formula for concordances (see Burghelea and Lashof [1, Section A] or Hatcher [3, App. I]), we may assume that $\operatorname{id}_{F \times I \times B}$ is isotopic to $H \times \mathrm{id}_{B} \operatorname{rel} F \times\{0\} \times B$ (if $\operatorname{dim}(F \times B)$ is sufficiently large). Thus, there exists a homeomorphism $\tilde{G}: F \times I \times B \times I \rightarrow F \times I \times B \times I$ such that: 
(1) $\tilde{G}$ is fiber preserving over the final $I$ factor,

(2) $G=\tilde{G} \mid: F \times\{1\} \times B \times I \rightarrow F \times\{1\} \times B \times I$ is an isotopy from $h \times \operatorname{id}_{B}$ to $\mathrm{id}_{F \times B}$,

(3) $H \times \operatorname{id}_{B}=\tilde{G} \mid F \times I \times B \times\{1\}$,

(4) $\tilde{G}$ is the identity on $F \times\{0\} \times B \times I \cup F \times I \times B \times\{0\}$.

It follows that for $0 \leq s \leq 1$, the map $F \times B \times I \rightarrow B \times I$ defined by

$$
(f, b, t) \mapsto \operatorname{proj} \circ \tilde{G}(f, s, b, t)
$$

is a homotopy rel $F \times B \times \partial I$ from proj: $F \times B \times I \rightarrow B \times I$ to proj $\circ G$ : $F \times B \times I \rightarrow B \times I$. Thus, $h$ and $G$ satisfy the hypotheses of Corollary 5.2.

When asphericity or stronger (curvature) conditions are placed on $M$, then more specific information about controlled homeomorphisms can be obtained as the next two results illustrate.

Corollary 5.4 Let $p: M \rightarrow B$ be a manifold approximate fibration with $\operatorname{dim} M \geq 5$ and $B$ a closed manifold of nonpositive curvature. If $M$ is aspherical, then the forgetful homomorphism

$$
\pi_{n} \operatorname{TOP}^{c}(p: M \rightarrow B) \longrightarrow \pi_{n} \operatorname{TOP}(M)
$$

is injective for $n \geq 1$ and split injective for $n \geq 2$.

Proof Combine Example 2.1 and Theorem 5.1.

When $M$ is also nonpositively curved, then Corollary 5.4 can be combined with the deep results of Farrell and Jones [2] concerning $\pi_{n} \operatorname{TOP}(M)$ in the stable range to give the following specific results.

Corollary 5.5 Let $p: M \rightarrow B$ be a manifold approximate fibration with $m=\operatorname{dim} M \geq 11$ and $M$ and $B$ closed manifolds of nonpositive curvature.

(1) If $2 \leq n \leq(m-7) / 3$ and $\hat{n}=[(n+4) / 2]$ !, then

$$
\pi_{n} \operatorname{TOP}^{c}(p: M \rightarrow B) \otimes \mathbb{Z}\left[\frac{1}{\hat{n}}\right]=0 .
$$

(2) If the center of $\pi_{1} M$ vanishes, then

$$
\pi_{1} \operatorname{TOP}^{c}(p: M \rightarrow B) \otimes \mathbb{Z}\left[\frac{1}{2}\right]=0 .
$$

Proof Combine Corollary 5.4 with Farrell and Jones [2, 3.7 and 3.8].

Finally, we point out that $\pi_{0} \operatorname{TOP}^{c}(p)$ is the domain of torsions of homeomorphisms $h: M \rightarrow M$ with the property that $p h$ is sufficiently close to $p$. This does not require a curvature condition on $B$.

Proposition 5.6 Let $p: M \rightarrow B$ be a manifold approximate fibration where $B$ is a closed manifold with a fixed metric $d$ and $\operatorname{dim} M \geq 5$. For every $\epsilon>0$ there exists a $\delta>0$ such that if $h: M \rightarrow M$ is a homeomorphism with $d(p h, p)<\delta$, then there exists an element $\tau_{\epsilon}(h) \in \pi_{0} \operatorname{TOP}^{c}(p)$ satisfying: 
(1) if $g, h: M \rightarrow M$ are two homeomorphisms with $d(p g, p)$ and $d(p h, p)$ both less than $\delta$, then $\tau_{\epsilon}(g)=\tau_{\epsilon}(h)$ if and only if there exists a $p^{-1}(\epsilon)$-isotopy from $g$ to $h$,

(2) if $h: M \times[0,1) \rightarrow M \times[0,1)$ is a controlled homeomorphism, then there exists $t_{0} \in[0,1)$ such that $\tau_{\epsilon}\left(h_{t}\right)$ is defined and equal to $[h] \in \pi_{0} \operatorname{TOP}^{c}(p)$ for all $t \geq t_{0}$.

Proof If $h: M \rightarrow M$ is a homeomorphism such that $p$ is sufficiently close to $p h$, then there exists a 1-parameter family of manifold approximate fibrations $f: M \times I \rightarrow B \times I$ from $p$ to $p h$ [4]. Now Controlled Straightening $[5,14.4]$ implies that there exists a fibre preserving homeomorphism $G: M \times I \times[0,1) \rightarrow M \times I \times[0,1)$ such that $G \mid(M \times I \times$ $\{0\}) \cup(M \times\{0\} \times[0,1))=\mathrm{id}$ and $f G_{t} \rightarrow p \times \operatorname{id}_{I}$ as $t \rightarrow 1\left(G_{t}=G \mid(M \times I \times\{t\})\right.$. Define $H: M \times[0,1) \rightarrow M \times[0,1)$ by $H(x, s)=\left(h \times \mathrm{id}_{[0,1)}\right) \circ G(x, 1, s)$. Then $H$ is a vertex of $\operatorname{TOP}^{c}(p)$ and we set $\tau_{\epsilon}(h)=[H] \in \pi_{0} \operatorname{TOP}^{c}(p)$. The properties follow from [4], [5, Section 14].

\section{References}

[1] D. Burghelea and R. Lashof, Geometric transfer and the homotopy type of the automorphism groups of a manifold. Trans. Amer. Math. Soc. 269(1982), 1-38.

[2] F. T. Farrell and L. E. Jones, Topological rigidity of compact nonpositively curved manifolds. In: Differential Geometry: Riemannian Geometry (eds. R. Greene and S. T. Yau), Proc. Sympos. Pure Math. 54(1993), Part III, 229-274.

[3] A. E. Hatcher, Concordance spaces, higher simple-homotopy theory, and applications. In: Algebraic and Geometric Topology (ed. R. J. Milgram), Proc. Sympos. Pure Math. 32(1978), Part I, 3-21.

[4] B. Hughes, Approximate fibrations on topological manifolds. Michigan Math. J. 26(1985), 167-183.

[5] B. Hughes, L. Taylor and B. Williams, Bundle theories for topological manifolds. Trans. Amer. Math. Soc. 319(1990), 1-65.

[6] Bounded homeomorphisms over Hadamard manifolds. Math. Scand. 73(1993), 161-176.

[7] Rigidity of fibrations over nonpositively curved manifolds. Topology 34(1995), 565-574

[8] Splitting forget control maps. In preparation.

$[9] \longrightarrow$ Controlled surgery theory over manifolds. In preparation.

[10] T. B. Rushing, Topological embeddings. Pure and Appl. Math. Series 52, Academic Press, New York, 1973.

[11] S. Weinberger The topological classification of stratified spaces. Chicago Lectures in Mathematics Series, Univ. of Chicago Press, Chicago, 1994.

Department of Mathematics Vanderbilt University

Nashville, Tennessee 37240

USA

email: hughes@math.vanderbilt.edu
Department of Mathematics

University of Notre Dame

Notre Dame, Indiana 46556

USA

email: taylor.2@nd.edu

Department of Mathematics

University of Notre Dame

Notre Dame, Indiana 46556

USA

email: williams.4@nd.edu 